\title{
Être en reste face aux résidus nucléaires
}

Being Left Over: Nuclear Remainders

\section{Yoann Moreau, Mathias Crémadez et Kyoko Maruyama}

\section{OpenEdition}

Journals

Édition électronique

URL : https://journals.openedition.org/tc/7816

DOI : $10.4000 /$ tc. 7816

ISSN : 1952-420X

\section{Éditeur}

Éditions de l'EHESS

\section{Édition imprimée}

Date de publication : 31 octobre 2016

Pagination : 92-109

ISBN : 9782713225291

ISSN : 0248-6016

Référence électronique

Yoann Moreau, Mathias Crémadez et Kyoko Maruyama, « Être en reste face aux résidus nucléaires », Techniques \& Culture [En ligne], 65-66 | 2016, mis en ligne le 31 octobre 2018, consulté le 29 septembre 2022. URL : http://journals.openedition.org/tc/7816 ; DOI : https://doi.org/10.4000/tc.7816 


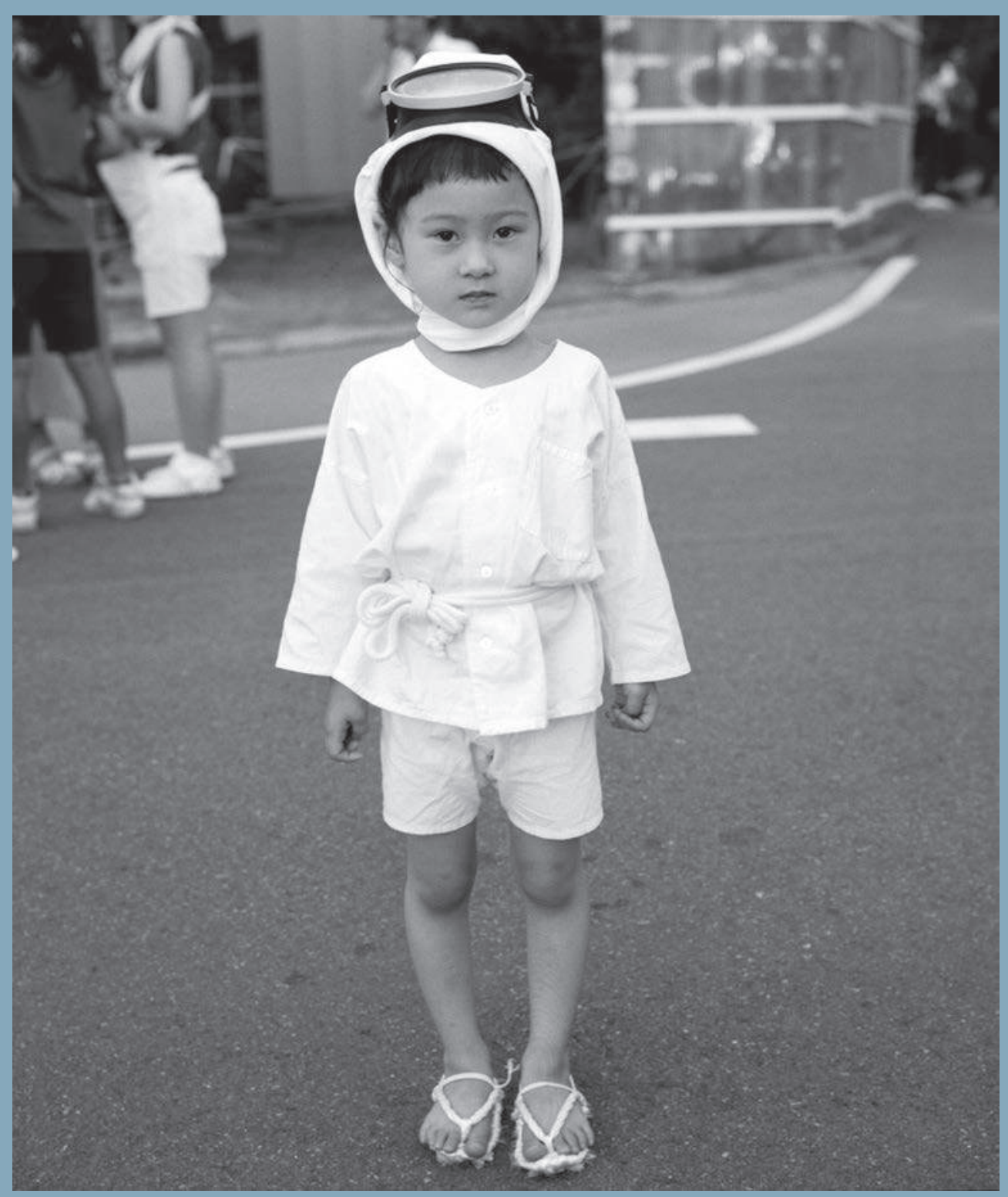




\title{
Être en reste face aux résidus nucléaires
}

\begin{abstract}
«Il se demandait ces derniers temps, chaque fois qu'il lisait des documents sur la vie des sauvages primitifs ou regardait des photos [...], s'il n'aurait pas pu lui aussi naître parmi eux. [...] Et combien d'objets invisibles, inaudibles ou impensables à présent [aurait-il] pu voir alors, et entendre et penser, [s'il] était né dans ces autres existences. Cette pensée-là avait quelque chose d'intolérable et d'effrayant, et en même temps d'intolérablement agaçant. Il y avait donc (c'était une question de faculté, non d'expérience) des choses qu'il ne pouvait ni voir ni entendre ni penser.»
\end{abstract}

Atsushi Nakajima ${ }^{1}$

\section{I'expérience des catastrophes}

Les catastrophes génèrent un vaste redéploiement du rapport humain à ce qui l'environne. Les survivants se retrouvent dans des situations qui déplacent considérablement leurs existences, bouleversant jusqu'aux usages les plus ordinaires. Une casserole pourra servir de marteau ou de casque, une bâche de couverture ou d'abri, un bus fera office de maison communautaire ou de matière première, pièces et déchets mis au rebut pourront s'avérer essentiels à la survie. En situation de désastre, la rationalité à l'œuvre chez le bricoleur prend le pas sur celle de l'ingénieur, la fabrique de fictions prévaut sur la recherche immédiate d'une objectivité historique. Ces logiques de la ruse et du détournement (par le bricolage, par la fiction) procèdent par «arrangements ». Elles opèrent selon une «logique du concret» qui sait composer avec les «moyens du bord», d'« indéfinissables débris » et «fragments [...] issus d'un processus de cassure et de destruction» (Lévi-Strauss 1962: 51). De fait, quand il s'agit de composer avec le désordre et la déréliction, elles s'avèrent plus pertinentes que les processus d'objectivation consistant à produire des «faits» et à reconduire un appareil technique et conceptuel préexistant. 
Au niveau sociologique, le fatras et le chaos engendrés réagencent également les sociétés. Les études qui portent l'attention sur la recomposition des mondes après bouleversement ont mis au jour les rouages pragmatiques et discursifs qui coordonnent la dispute entre le paradigme dominant et les rationalités jusque-là en coulisses de la scène sociale. Ces reconstructions sont complexes, qui opèrent par disputes sociales et controverses (Revet 2007; Chateauraynaud 2011; Glowczewski \& Soucaille 2011; Moreau 2013). Aucun déplacement ne va se fonder sur l'exception, celle-ci tendant par définition à confirmer la règle: ce n'est pas parce qu'une casserole peut servir à planter des clous que cet usage devra être généralisé à l'ensemble des contextes. Il faut que l'exception ait un caractère irréductible qui puisse justifier de «faire avec » sur le long terme.

Tenter d'intégrer ce qui a été subi passe par une «reconnaissance» (Honneth 2006), c'est-àdire par l'acceptation d'une forme d'altérité qui échappe à la maîtrise, au contrôle, à la domestication. Quelle que soit l'origine imputée de ce qui a été subi (géologique, météorologique et biologique, ou humaine), sa reconnaissance sur le plan social et institutionnel est une affaire délicate. C'est une chose de reconnaître le caractère existentiel du risque sismique, climatique ou épidémiologique; c'en est une autre de lui adjoindre une menace supplémentaire, par exemple nucléaire, écologique ou terroriste. Admettre un nouveau registre de négativité accroît le sentiment d'inconfort et d'incertitude, étend la part de signification flottante et d'insatisfaction qui peut parfois conduire à dresser un constat pragmatique fataliste: «Tout n'est pas rose, c'est ainsi, et on n'y peut rien changer.»

Mais, dans la mesure où ces événements stimulent «le travail du négatif » (Laplantine 2015), ils ne sont pas saisis dans l'horizon d'un hasard pur, dont on ne pourrait ni infléchir ni circonscrire la contingence. Dans la mesure où à la déception et au dépit répondent non pas une désaffection et une «indolence» existentielle (Anders 2008), mais une forme active de mobilisation individuelle et de transformation du champ social, le surgissement du désordre ne relève pas d'une forme de fatalité qui consisterait à le subir sans réagir. Certes l'épreuve de la catastrophe contraint les individus et les collectifs qu'ils composent à adapter leurs modes d'existence, mais aussi à susciter des inflexions culturelles et sociales inédites. Des relations de voisinage peuvent être initiées puis engagées sur le très long terme, formant des collectifs bien au-delà de l'effervescence de l'évènement; certaines fictions traumatiques vont participer à la création de nouvelles figures de l'imaginaire et de la littérature et, ce faisant, influer sur les horizons et les modes d'existence; certains camps de réfugiés bricolés dans l'urgence vont se maintenir plusieurs décennies, installant de facto des modes d'administration et de vie collective en marges de la culture dominante.

Il convient d'être vigilant à l'égard d'une forte propension au catastrophisme. Celui-ci tend à étudier la réparation des mondes post-catastrophes en ayant pour focale des «victimes » envisagées dans l'optique passive (parce que subie) d'une résilience et d'une adaptation. Incontestablement, sous l'effet d'un choc violent un individu est tenu de s'adapter (revoir par le bas ses besoins et ses envies en fonction des contraintes qui s'imposent) et d'être résilient (se recomposer tout en gardant la trace indélébile d'une zone de fragilité et de vulnérabilité). Mais des formes plus volontaires de recomposition, par exemple sous la forme d'une co-suscitation (Berque 2014), sont également mises en branle. Une catastrophe initie également des 
dynamiques novatrices, conduit à développer d'autres champs de compétences et de relations, active des capacités jusque-là ataviques ou dédiées à d'autres fonctions. Ces situations provoquent et révèlent de nombreuses «prises» (Berque 2000) et «affordances» (Gibson 1979) inédites, qui engagent les êtres et les choses dans de nouveaux rapports, récits et pratiques.

Ainsi, les manières de refaire monde après un bouleversement demeurent ambivalentes. Elles sont à la fois subies et volontaires, passives et actives, destructrices et fondatrices. Comme tout handicap, elles n'affectent pas l'ensemble des registres d'expression et de compétence, mais incitent aussi à amplifier et manifester d'autres qualités; comme tout phénomène anormal, elles ne sont pas nécessairement et uniquement pathologiques, mais peuvent participer au maintien d'un équilibre plus général (Canguilhem 2006 [1943]); comme tout événement, elles engagent une pluralité de protagonistes et d'interprétations, ce qui a été vécu comme catastrophique par certains pouvant relever d'une stratégie volontaire et désirée par d'autres (Moreau 2014). Cette ambivalence radicale, qui donne lieu à disputes et controverses, caractérise les catastrophes. Pouvoir la décrire appelle un registre de connaissance particulier, acquis ex-periculum, c'est-à-dire «par le truchement d'une épreuve, d'un péril ». C'est précisément l'étymologie du terme expérience (Rey 2004), une connaissance acquise au travers de quelque chose qui a été vécu sur le mode du subir. En ce sens, l'expérience rejoint son acception scientifique ordinaire. Elle permet de découvrir et d'asseoir de l'inédit, des nouveaux corpuscules ou des nouveaux modes de sociabilités et de pratiques, de nouvelles manières d'ausculter la matière et les lois qui la régissent, ou de se raconter et de se projeter dans l'avenir. De ce point de vue, toute expérience est reconnaissance d'une résistance (du négatif), qu'elle soit provoquée (la preuve expérimentale) ou subie (l'expérience d'une catastrophe).

\section{Une culture du subir}

Le négatif-la mort, la souffrance, l'injustice, les déchets-motive des raisonnements et des usages en marge des pratiques, des modes d'objectivation et des rationalités ordinaires. L'expression «culture du subir» désignera ici l'ensemble des manières au moyen desquelles des individus composent activement avec ce qu'ils ne maîtrisent et ne désirent pas. Elle traduit l'existence incompressible d'une «dimension subie» (Laplantine 2015) qui contraint les collectifs à tenir compte du négatif.

La culture du subir ne relève pas de l'exception anthropologique. Les processus de deuil consistent précisément à reconnaître une négativité originaire incompressible. Les sépultures procèdent de cette volonté de maintenir le souvenir de ce qui a été perdu et de ceux qui ont disparu ; le statut de défunt distinguera cadavre humain et déchet. Les mémoriaux élaborés en souvenir des victimes des grandes guerres, les stèles et monuments dédiés aux grandes catastrophes et aux événements tragiques vont également dans le sens du maintien d'un principe de réalité qui met en défaut et fait surgir des situations de déprise. Mémoriaux, stèles, monuments et sépultures activent et entretiennent un «devoir de mémoire» (Ricœur 2000), préservent 


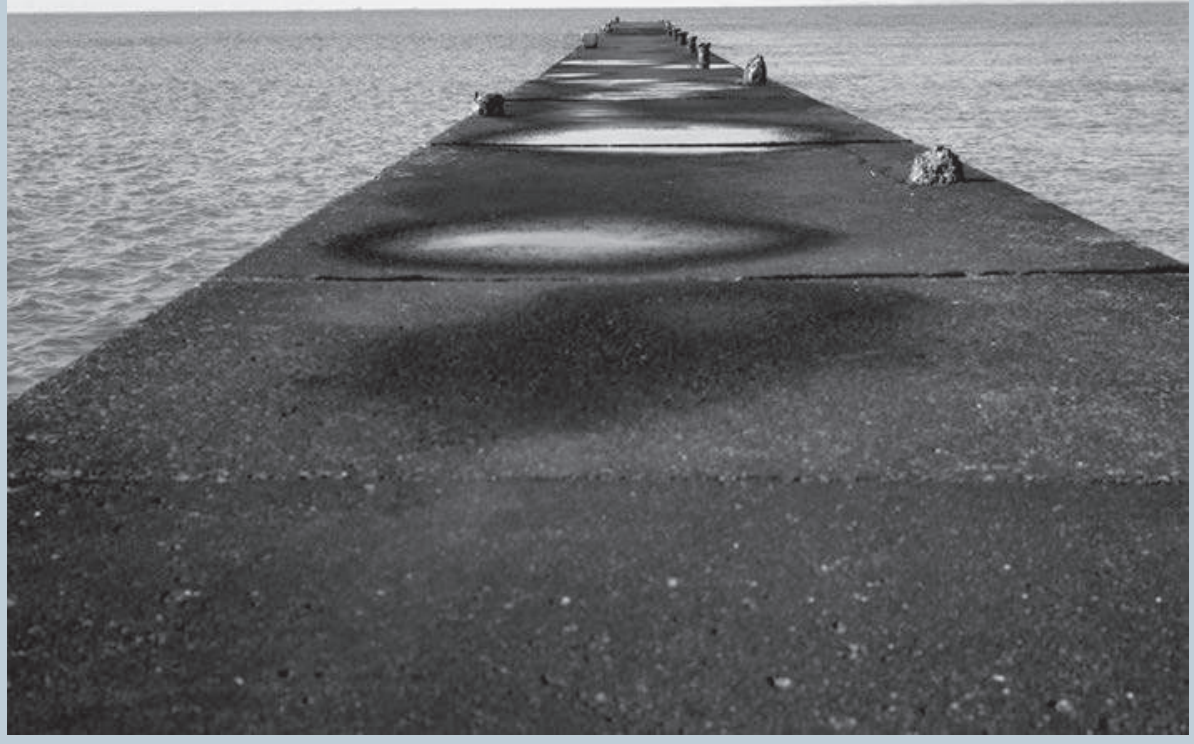





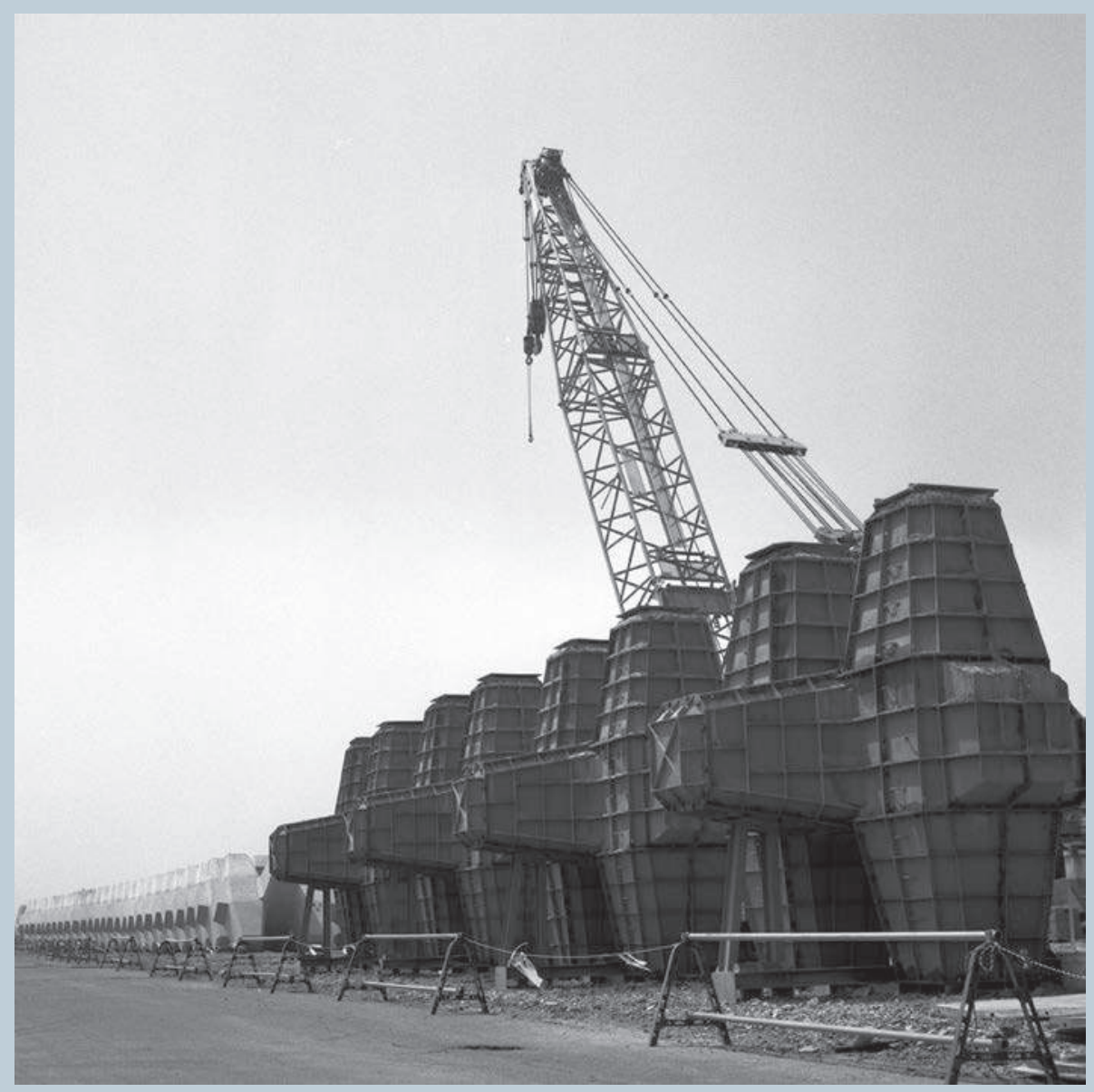




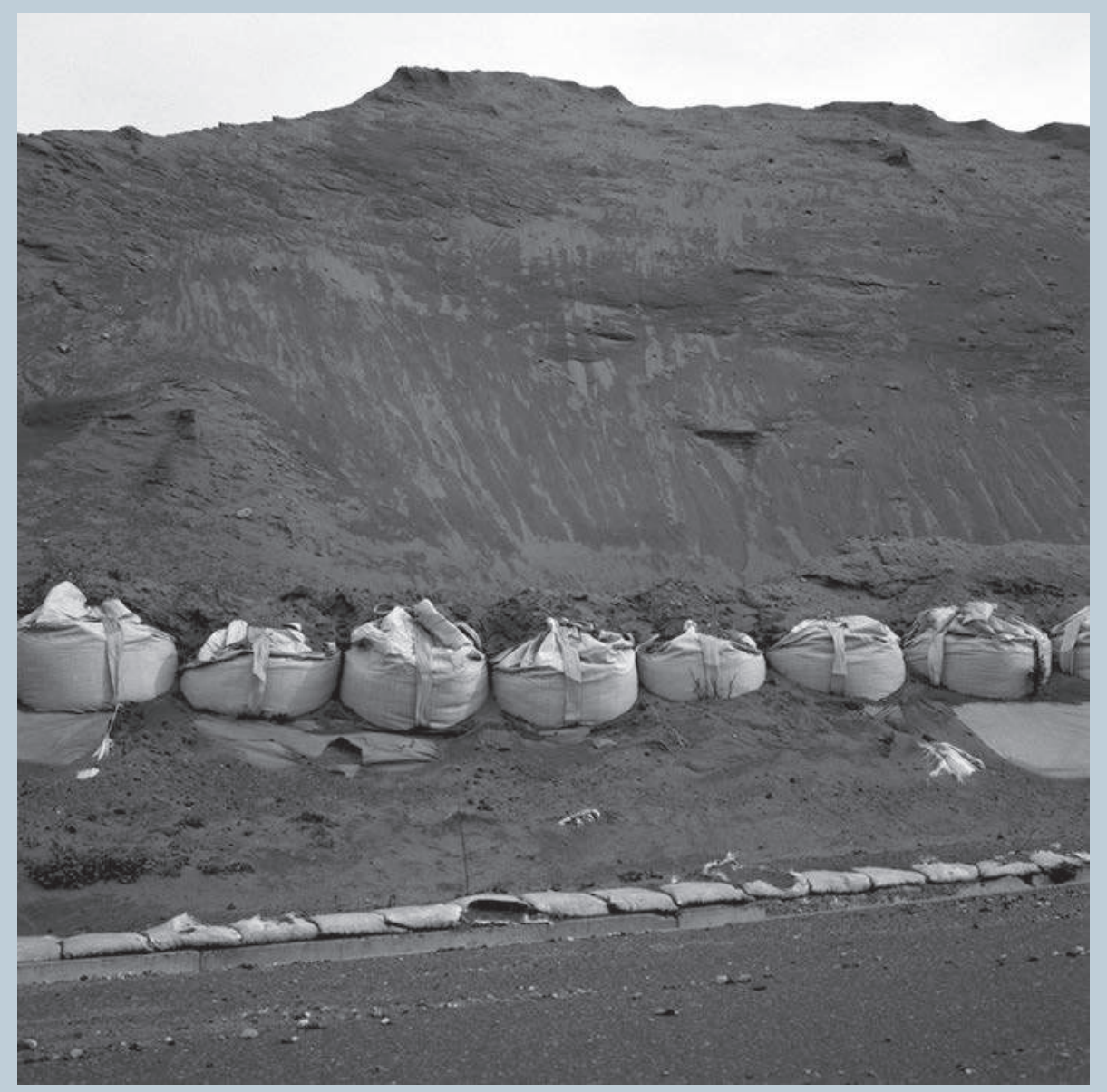


l'indice de ce qui a eu lieu par le passé, signent les limites de l'agir humain, tracent les contours de ce qui excède sa capacité de maîtrise. Ainsi, bien que les individus tendent à se souvenir des choses agréables, il leur arrive aussi, dans certains cas, de cultiver le souvenir d'une perte, d'un échec, d'une déprise, d'une souffrance ou d'un événement catastrophique. Ces productions maintiennent le souvenir d'une expérience éprouvante par émergence d'une singularité sociale, par exemple sous l'espèce du sacré et du consacré.

Les raisons et principes de cette émergence sont difficiles à cerner, le négatif étant plutôt susceptible d'être forclos, ou «exclu», du dispositif culturel (Foucault 1972). Cette question a profondément travaillé les philosophes modernes, collectivement hantés par ce qui a surgi au travers de la Shoah et d'Hiroshima. La nécessité de devoir composer avec une «Part maudite» (Bataille 1967) peut engager de l'« imprescriptible» (Jankélévitch 1986). L'engagement est alors d'ordre éthique, manière d'exprimer que cette prise de position ne peut pas être fondée sur un ordre préétabli (moral ou politique), mais repose nécessairement sur la reconnaissance d'un désordre historique ("ça a eu lieu»). Parce qu'une industrialisation de la mort humaine et l'explosion de bombes nucléaires se sont produites dans l'histoire, cela peut resurgir malgré nous et hors de tout contrôle, comme les guerres «classiques» et les séismes.

Au niveau du vécu ordinaire, la «mise en culture» des défunts, notamment leur traitement aux moyens de rituels (funérailles, inhumation, crémation, traitements des cadavres), de moments (jours consacrés, veillées) et de sites socialement dédiés (cimetières, autels domestiques, tombes), permet de maintenir la séparation entre les vivants et les morts. La prise en charge des dépouilles ambitionne aussi de préserver le collectif des vivants des tourments d'un deuil inachevable, des fantômes et autres spectres d'un passé qui ne peut jamais tout à fait être dépassé (Despret 2015). Le mort ouvre et permet la reconnaissance de la mort (Guy et al. 2014; Souffron 2015). L'existence de dispositifs sociaux dédiés au deuil est l'une des expressions de la «culture du subir». Ils s'appliquent à ce qui a été subi, est subi, et sera encore à subir, à une expérience-épreuve (la mort d’un proche) qui concerne toutes les générations, comme les aléas nucléaires.

\section{Le déchet et le neutre}

La production de matières radioactives étant liée à des enjeux majeurs (tant énergétiques que médicaux, militaires, géopolitiques et scientifiques), il semble inéluctable que leur amoncellement se poursuive sur le temps long de notre modalité civilisationnelle. Il y a bien accumulation parce que leurs périodes d'activité - notamment celles de certains isotopes de l'uranium et du plutonium - sont supérieures à la centaine de milliers d'années. Ces matières doivent donc pouvoir faire l'objet d'une prise en charge pérenne, robuste dans le temps. À défaut, leur existence risquerait de basculer progressivement de leur qualification en tant que déchet, à leur disqualification sous la forme du neutre. Le neutre sera entendu ici comme ce qui signe l'absence de rapport, comme ce qui est oublié ou sans qualité et sans prise. Il qualifie précisément ce qui est 
disqualifié, «tout ce qui déjoue le paradigme» (Barthes 2002: 31). Il s'agit de ce qui échappe à la reconnaissance (l'Autre) et à la perception (la Nuit, au sens de Blanchot), à l'agir technique (l'inutilisé) et symbolique (l'inqualifié). Mais alors, comment parler du neutre, si rien ne nous relie à lui? Il nous est peut-être possible de le cerner «par défaut».

L'éthologue Jacob von Uexküll (1864-1944) a montré que l'environnement regorge d'éléments neutres, car, pour chaque forme de vie, seuls certains aspects environnementaux sont connotés, c'est-à-dire pris dans un rapport de coexistence et envisagés avec l'horizon d'un usage et d'une signification (Uexküll \& Kriszat 1956 [1934]). Entre Umgebung (environnement) et Umwelt (milieu) il y a « un reste» non connoté qui échappe à l'écologie symbolique (la connaissance), technique (l'action) et organique (la perception) ${ }^{2}$. Ce «reste» n'est pas identique d'une espèce à l'autre. Si pour une tique le son d'une sirène relève du neutre, qu'elle ne le perçoit ni n'interagit avec lui d'aucune façon, il n'en va pas de même pour un chien ou un humain qui le connotent chacun à sa manière, selon son dressage ou son éducation. Réciproquement, la tique sera sensible à des aspects de l'environnement que le chien et l'humain ignorent.

Toutefois, certains éléments de l'environnement jouent le rôle de substrat de signification commun, par exemple certaines gammes de fréquence sonore ou visuelle sont partagées: le chien reconnaît la voix de son maître et distingue certains sons ("Médor», "couché », "va chercher », etc.). Réciproquement, le maître connote les comportements de son chien (quand il remue la queue, baisse les oreilles, retrousse les babines, aboie ou gémit). Ce rapport «de contrepoint», dit Uexküll, c'est-à-dire de recouvrement partiel des significations et des champs d'organicité, opère également au niveau de l'écologie matérielle. Les résidus organiques participent d'un système d'échange inter-spécifique au sein duquel les déjections d'une espèce constituent les aliments d'une autre. Ce qui est déchet et risque pour l'une peut être ressource et agrément pour une autre. Ainsi, un trognon de pomme est un déchet pour l'humain, mais demeure «signifiant » pour d'autres espèces (par exemple pour les fourmis).

Convenons d'appeler restes ${ }^{3}$ ce qui ne relève pas de cette écologie matérielle, c'est-à-dire ce pour quoi il n'existe pas de contrepoint qui permette un réemploi, une transformation et, à terme, un «cyclage». Pour le dire dans le langage d'Uexküll, un reste est ce qui est sans contrepoint, non connoté par les autres espèces, c'est-à-dire neutre: sans usage, ni valeur, ni fonction.

\section{Voir et savoir, une fracture empirique}

Dans l'état actuel de nos connaissances, les résidus nucléaires ne servent à aucune forme de vie et aucune espèce n'est biologiquement constituée pour les percevoir. Ce sont donc des restes (et non de simples déchets), des restes humains. S'il convient de préciser ici l'aspect anthropologique des résidus nucléaires, ce n'est pas tant pour souligner le rôle que cette espèce joue dans leur production, mais parce que, de toutes les espèces, l'humain est le seul pour qui les rayonnements ionisants ne sont pas neutres. Lui seul connote les radiations $\alpha, \beta$ et $\gamma$ émises par les 


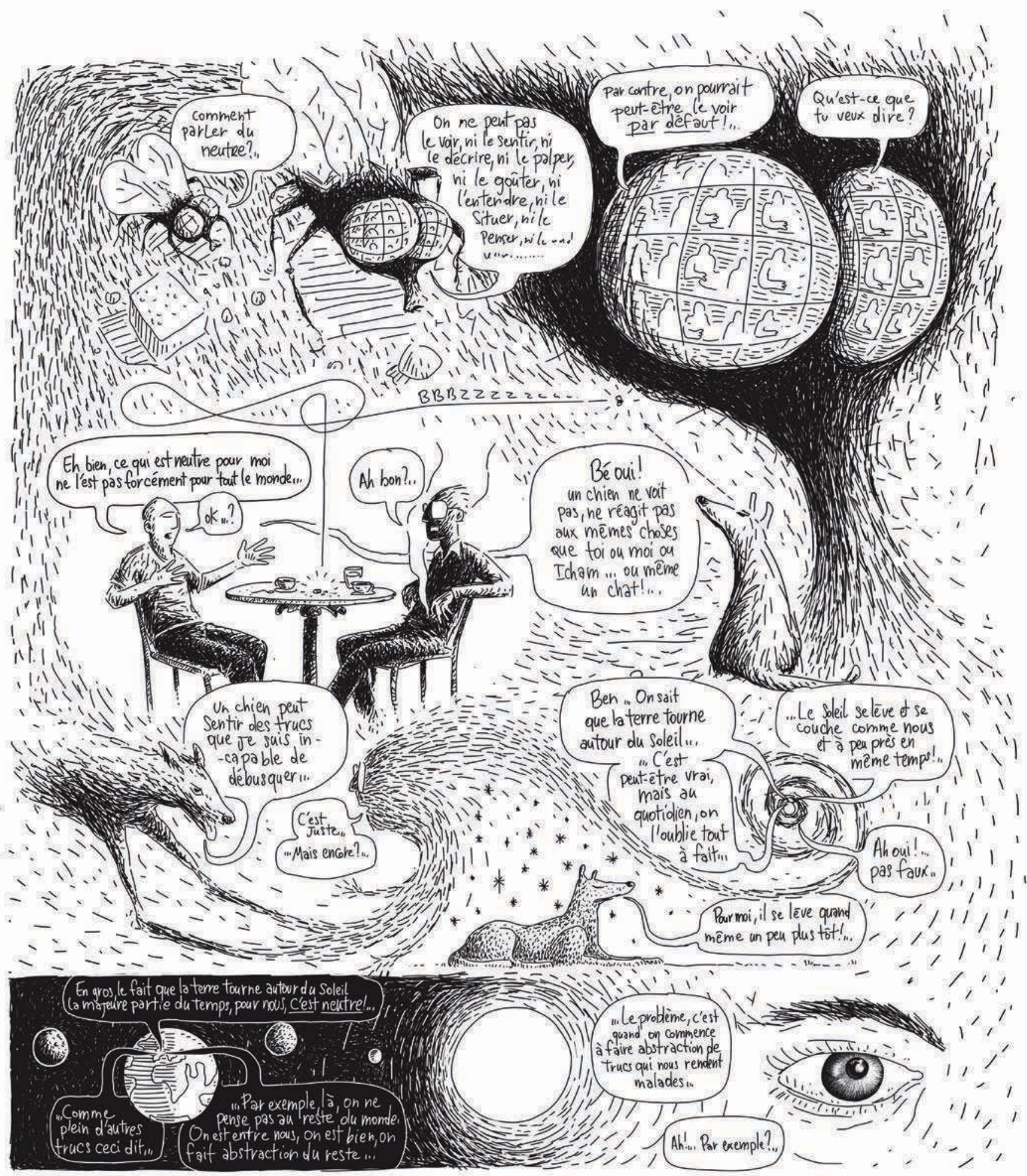




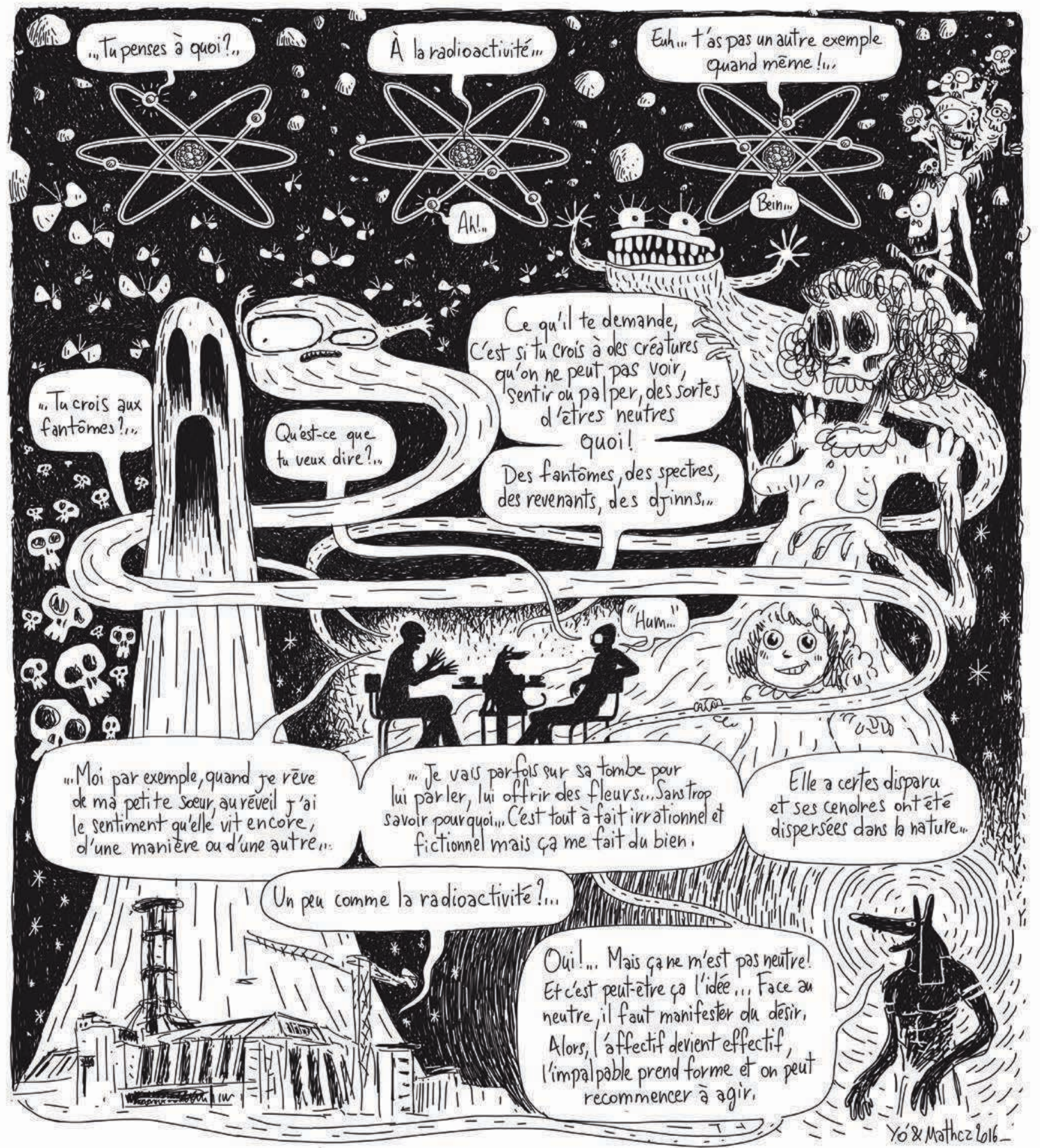


isotopes atomiques; lui seul est appareillé pour avoir connaissance de ce qui palpite dans des phénomènes invisibles, insipides, inodores, impalpables et silencieux; lui seul peut établir un lien entre ces rayonnements et certaines des pathologies organiques qui affectent les vivants. Cet aspect de la relation humaine à ce qui l'environne est fondamental pour comprendre notre rapport à la radioactivité: nous savons qu'elle existe et qu'elle constitue un stress organique, bien qu'elle ne se traduise pas en sensations. Si, à la différence des autres espèces animales, nous avons déserté les alentours de Tchernobyl et de Fukushima, c'est parce que nous savons que ces régions sont contaminées et pouvons le mesurer, par exemple au moyen de compteurs Geiger. De nos jours, pour peu qu'il ait eu vent des accidents nucléaires, chacun se sait vivre sous faibles doses ionisantes.

Connotés scientifiquement mais neutres à l'échelle de la sensibilité organique, ces rayonnements soulèvent des problèmes similaires à ce qui motiva les instigateurs de la phénoménologie. Pour Husserl, la vérité scientifique d'une Terre en perpétuel mouvement ne pouvait se substituer à l'authenticité phénoménologique d'une planète qui apparaît comme immobile et stable. Pour le philosophe, il convenait donc de réopérer un « couplage » entre ces deux pans de l'expérience humaine, au risque, sinon, de perdre pied avec la réalité concrète (Husserl 1989 [1934]).

Ce type d'embrayage est bien plus fréquent qu'on ne le croit. Par exemple, pour le physicien, le Soleil brille tout le temps mais, pour l'humanité qui arpente les contrées terrestres, la nuit est obscure et le jour lumineux. Ces deux propositions - «le Soleil brille nuit et jour » et « le soleil brille le jour mais pas la nuit »-correspondent à deux régimes de vérité 4 . La première relève d'un savoir acquis et constitué, la seconde est relative au vécu ordinaire et quotidien. Ces deux perspectives correspondent à deux niveaux du savoir, celui de l'astronome ou de l'allumeur de réverbère, de l'expérimentation ou de l'expérience. Elles sont certes en contradiction mais ne soulèvent pas le sentiment d'une aporie existentielle. Le fait que nous soyons capables de tenir ensemble ce qui se contredit, «le Soleil brille jour et nuit » et «le soleil brille le jour, mais pas la nuit», signe que nous privilégions le concret. Ce concret, toujours complexe, prévaut sur l'objectivité pure (d'un astre en combustion perpétuelle) ou la subjectivité radicale (d’un astre qui «se lève» et «se couche»).

\section{Vers une " couture " de la fission}

Le monde avec lequel nous interagissons concrètement conjoint une part sensible (saisie et ressentie dans l'instant) et une part sensée (qui convoque des choses apprises auparavant). Le « couplage», ou plutôt la « couture» (Montaigne 1979 [1588]) entre ces deux foyers cognitifs est délicate à saisir ${ }^{5}$. Elle engage en effet deux dimensions. La première couture, d'ordre ontologique, met en tension le dualisme, qui distingue matière et pensée, quantitatif et qualitatif, nature et culture ${ }^{6}$. La seconde, d'ordre temporel, sous-tend le monisme, qui confond en une seule substance et sans distinction de niveaux, réalité des lois de la physique et réalité historique, monde «céleste» et «sublunaire», structures et systèmes. Dualisme et monisme sont 
deux accidents possibles de cette indispensable couture cognitive: sa déchirure (le dualisme) ou sa disparition dans l'étoffe (le monisme).

Le «Grand Partage» dualiste prend l'eau et se fissure de toutes parts (Descola 2005), en revanche la critique de ce que nous allons qualifier de «Petit mélange» est nettement moins à l'ordre du jour, et, du coup, moins aisée. Le «Petit mélange» est cette tendance à l'accoutumance, à la banalisation, à la naturalisation, à la renormalisation progressive de toute nouveauté. Husserl la nomme «habitualisation » ou «sédimentation», Blanchot l'exprimera en posant que «le non-familier se définit comme ce qui a vocation à devenir familier» (Zarader 2001 : 96). Ces accidents cognitifs - «Grand Partage » et «Petit mélange »-introduisent une perte de sens du concret. Cela provoque, d'une part, un daltonisme généralisé par recours systématique à des catégories duales qui découplent monde sensible et savoirs acquis et, d'autre part, un affadissement existentiel par usure d'un régime d'historicité réduit au présentisme (Hartog 2003).

La radioactivité, invisible et durable, touche «par les deux bouts» au caractère concret du vécu des personnes, affectant conjointement son aptitude sensible et sa capacité mémorielle. Elle tend ainsi à accentuer ces deux biais cognitifs: insensible mais sensée, elle renforce l'expérience dualiste du monde; événement voué à perdurer sur le très long terme, elle ne fait exception que dans l'horizon d'une banalisation. Préserver l'échelle concrète de l'existence humaine est l'un des problèmes que pose la production de matières instables au niveau atomique. La manière dont nous allons qualifier, nommer et traiter les résidus nucléaires participe de la fabrique de la couture-culture d'une fission qui affecte à la fois les noyaux de la matière et la cognition humaine.

\section{Pour une culture de la fission}

Ce qui fait défaut à notre prise en charge, pour l'heure majoritairement techniciste, est d'ordre symbolique et temporel. Afin d'éviter les défections cognitives introduites par la fission de l'atome (le «Petit Mélange» de la banalisation et le «Grand Partage» du dualisme), il conviendrait: a) de déployer une terminologie qui tient compte des spécificités des résidus atomiques (que gomme la notion de déchets), par exemple au moyen de la notion de « restes »; b) d'accroître la robustesse temporelle des dispositifs sociaux que nous déployons à l'égard des rayonnements ionisants, au risque, sinon, de fabriquer du neutre.

Concernant ce deuxième point, nous pourrions avoir recours aux outils performatifs de la «culture du subir». Ses appareils symboliques et techniques en marges de la rationalité dominante (notamment rituels, récits à ancrage mythologique et «bricolages») élaborent des ruses épistémiques face au processus de «neutralisation de l'histoire» (Reitz 2011: 96). Ils mettent en culture des dispositifs qui visent à rappeler ce qui a provoqué, provoque et provoquera, une déprise. Ils reportent continuellement la banalisation en maintenant actif le « désir du neutre » (Barthes 2002). Cela passe par des processus d'augmentation de la réalité, par exemple en adjoignant à la disparition de la matière (le corps d'un être cher, la destruction d'un lieu de vie) une 
charge immatérielle (du spectral, du mémoriel, de l'agentivité). Le désordre ne sera alors plus envisagé comme un simple fatras de choses, mais comme un mélange des catégories provoquant une souillure (Douglas 1971) contenant le germe d'une transmission-contamination du champ symbolique (Zonabend 1989: 183).

À bien y regarder, cette mobilisation du mode culturel à l'œuvre dans les situations subies est peut-être déjà en train d'émerger dans le traitement non académique de Tchernobyl et Fukushima. Une partie du vocabulaire utilisé pour décrire les grandes catastrophes atomiques semble en effet esquisser une forme de résistance à cette mouvance structurellement neutralisatrice.

Pour décrire les structures d'acier et de béton fabriquées pour minimiser les fuites des réacteurs de Tchernobyl et de Fukushima Daichi, le champ sémantique n'est pas spécifiquement technique. Il contient des mots dont la charge symbolique est considérable. Nommer ces enceintes de confinement «sarcophage» ou «Arche» véhicule le potentiel d'une mobilisation concrète qui surmonte les affres du «Grand Partage» (qui sépare le matériel et le symbolique) et du «Petit Mélange» (qui écrase les temporalités). Le premier terme, usuellement employé pour définir le tombeau d'un pharaon momifié, indique le lieu d'une dépouille supputée immortelle; le second renvoie au Déluge biblique. Ces mots - «sarcophage» et «Arche»-, parce qu'ils confèrent à ces structures une existence à la fois matérielle et symbolique et s'inscrivent dans des régimes de temporalités longues, semblent répondre à la menace de coupure et d'affadissement cognitifs que nous avons relevée. Dans une veine similaire, qualifier de «sacrifice» l'envoi des premiers contingents d'intervention mobilisés sur les sites «souillés» de Tchernobyl et de Fukushima traduit une expérience qui déborde la seule réalité physico-chimique (Chouzenoux, Gorgerat \& Moreau 2013). Sarcophage, Arche, sacrifice et souillure, quatre termes qui mobilisent des modalités de prise en charge relevant, pour partie, des paradigmes d'une «culture du subir» en marge de la rationalité dominante.

Actuellement, la rationalité classique préconise uniquement de «stocker» et d'« entreposer» les résidus atomiques, termes qui correspondent au champ sémantique des déchets, et non des restes (en effet, si l'on «stocke» et «entrepose», c'est en vue d'un réemploi et d'une réutilisation). Sur ce point la posture d'une culture de la déprise serait tout autre, consistant à admettre que l'on ne peut simplement enterrer les restes nucléaires et que, de ce fait, afin d'éviter leur retour possible dans le monde des vivants, il conviendrait, par exemple, de procéder à leur enterrement. À tout le moins, elle viserait à composer sur le long terme en prenant en considération le risque de neutralisation symbolique des sites dédiés et des matières dispersées. Le traitement actuellement préconisé apparaît comme partiel, en reste d'une prise concrète, c'est-à-dire prenant en compte à la fois les dimensions physico-chimique et symbolique. Il fait l'impasse sur le rôle de la fonction symbolique dans le maintien des processus techniques. À défaut, si la perspective demeure techniciste, les structures de ciment qui confinent les cœurs des centrales de Tchernobyl et de Fukushima ne pourront faire l'objet que d'une maintenance (technique et protocolaire), et non d'un entretien (technique et symbolique, protocolaire et rituel). Or les menaces qui pèsent sur l'effritement progressif des sarcophages de Tchernobyl et de Fukushima, mais aussi sur les autres sites d'entreposage des résidus nucléaires, nous engagent à penser le très long terme. Il convient donc de réfléchir aux modalités culturelles aptes à suivre leur devenir sur les temps longs de l'histoire. 


\section{Le reste et la parole, proposition pour une couture de la culture du nucléaire}

Sur les temps longs de l'histoire, ceux que considère André Leroi-Gourhan dans Le geste et la parole, l'espèce humaine poursuit un processus d'extériorisation du corps animal en systèmes techniques - par des gestes - et d'introjection du registre technique en systèmes symboliques - par des paroles (Leroi-Gourhan 1964). Sur la question des résidus nucléaires, les prises en charge actuellement préconisées ne réalisent que la moitié de cette trajectoire: elle place les individus «en reste» d'une couture cognitive.

Pour le dire autrement, avec le vocabulaire du géographe Augustin Berque, sur les temps longs de l'histoire, nous habitons le monde, faisant de l'environnement (Umgebung) un milieu (Umwelt), mais, à défaut d'un processus de «trajection» (qui conjoint, par va-et-vient des processus d'objectivation et de subjectivation, des gestes et des paroles, des choses sensées et des choses sensibles, des substances et des prédicats), nous allons à contre-courant de cette mouvance longue des espèces. Cette tendance à perdre pied, le «métabasisme» (Berque 2000), et à exclure les temps longs, le «présentisme» (Hartog 2003), ne conduit plus de l'environnement vers la constitution d'un milieu. Bien au contraire, elle tend à aller du milieu existant vers l'environnement, c'est-à-dire dans le sens d'une déprise (technique), d'une anesthésie (des sensations) et d'une neutralisation (des choses signifiantes), bref, dans l'horizon d'une perte de sens quant à la manière dont nous faisons monde de ce qui nous arrive.

Infléchir cette tendance passe, c'est notre hypothèse, par une «reconnaissance». Les résidus nucléaires accentuent une déprise singulière qui ne peut pas être considérée comme simplement «accidentelle». Ils relèvent d'une modalité qui permet et agence notre rapport au monde, au long d'un processus voué à perdurer. Or, pour l'heure, nous ne savons comment maîtriser ce processus, ni le traiter techniquement, ni s'en passer socialement. Il convient, comme face à tout aléa, de préserver le «sens du concret ». Cela passe par une culture de ce qui tend à être neutralisé au sein du paradigme moderne classique. Une «couture» de ce paradigme de la maîtrise avec les opérateurs à l'œuvre dans la «culture du subir» semble de mise. Il s'agirait alors d'associer aux gestes purement techniques du traitement des restes radioactifs et de la maintenance des sites dédiés, des opérations visant à charger symboliquement ces matières et les lieux où elles reposent. L'ajustement du vocable est une première étape de composition d'un langage commun permettant un «contrerolle» (Montaigne 1979 [1588]) entre sciences et arts, objectivation et subjectivation, geste et parole, sensé et sensible, neutre et connoté. À défaut, si l'on demeure en reste d'une «culture de la couture», nous risquons d'accentuer encore, s'il en était besoin, la déchirure et l'affadissement du sens du concret. 


\section{Notes}

1. A. Nakajima, Le Mal du loup (1942), Paris, Allia, 2012: 30-31.

2. Uexküll a montré que ce « filtrage» d'une partie de ce qui nous environne est commun à toute forme de vie, mais il est aussi relatif à un mode culturel historiquement situé. Par exemple, le pétrole, matière organique engoncée depuis plusieurs millions d'années dans les sous-sols terrestres, est devenu au Xvinle siècle une source majeure d'énergie. De déchet fossile ne participant plus au cycle des matières carbonées de la biosphère, ce résidu organique est devenu, du fait de l'invention de nouvelles technologies et de nouveaux usages, une ressource de premier plan. L'invention du moteur à explosion a permis de démultiplier la connotation du pétrole, c'est-à-dire de lui conférer une existence concrète pour un grand nombre d'individus. C'est l'invention d'un certain type de technologie qui a découvert (au sens littéral, c'est-à-dire qui a «levé le voilesur ») la valeur et les usages possibles de l'élément géologique «pétrole» (Berque 2000).
3. La langue japonaise distingue nokori (残り, les restes), de nagori (名残, les vestiges, litt. «les traces de noms»), qui lui-même viendrait de nagori 余波 (litt. «les traces d'une vague »). La langue française n'opérant pas cette distinction, j'aurais tendance à traduire ce que j'entends par reste au moyen du son «nagori », exprimant à la fois le sillage du souffle (le langage) et de l'eau (les vagues).

4. Deux régimes de vérité qu'indique la distinction entre le Soleil (avec une majuscule, c'est-à-dire l'étoile) et le soleil (avec une minuscule, c'est-à-dire l'agent de la lumière pour les terriens).

5. Une véritable constellation sémantique nimbe cette idée de «couture» : « couplage» (Husserl), rapport «dialogique» (Morin, Bakhtine), «trajection» (Berque), «médiation» (Descola), connaissance «vibratoire» (Laplantine).

6. Ce clivage peut également être décrit en termes de «physicalité» et «intériorité» (Descola 2005); de «corps animal» et de «corps social» (Leroi-Gourhan 1964); de «part physiologique» et de «part médiale» (Berque 2000).

\section{Les auteurs}

Mathias Crémadez est illustrateur. Après avoir passé plusieurs années au Brésil, il vit et travaille actuellement à Paris. http://mathcz.tumblr.com.

Kyoko Maruyama est née à Fukushima. Depuis cinq ans, elle a développé un grand intérêt pour la technique et l'expression photographique. Après des études dans l'atelier de Satoru Wanatabe, elle a rejoint l'atelier de Osamu Moriya pour expérimenter l'art de la photographie de Noriko Kaneko. Elle est photographe depuis trois ans. Elle est représentée par la galerie Antonin Borgeaud (YPF Gallery).

Yoann Moreau est anthropologue, maître assistant au CRC (Mines ParisTech), chercheur associé à l'IIAC-Centre Edgar Morin (EHESS/CNRS). Ses recherches portent sur les catastrophes, les modes d'objectivation et la dramaturgie des connaissances. Il a récemment dirigé le numéro 96 de la revue Communications: Vivre les catastrophes (Seuil, 2015), et participe, en tant que dramaturge et performeur, à la création théâtrale (Médée/Fukushima, 2012; Manger Seul, 2014; Blanche/Katrina, 2016).

\section{Iconogaphie}

Image d'ouverture. Jeune fille vêtue à la manière des pêcheuses de perles en apnée (ama-san 海女さん) (c) Created by Kyoko Maruyama. Texte: Yoann Moreau. 1 à 4. Au long des rives de la préfecture de Chiba (à l'est de Tokyo), région affectée par le tsunami du 11 mars 2011 : 1. Une jetée, dont on ne sais si elle progresse dans l'océan par-delà le rivage, si elle se glisse sous le ciel Pacifique, ou si elle flotte entre les deux. Peut-être ni l'un ni l'autre.
2. Un panneau rappelle aux promeneurs que le ramassage des fruits de mer est autorisé, mais avec parcimonie, par respect pour les professionnels qui en vivent.

3. Provisoirement stockés en retrait des digues, chaque bloc de cette nouvelle génération de tétrapodes (消波ブロック) pèse suffisamment lourd (8 tonnes pour 3 mètres de hauts) pour ne pas être bringueballé comme fétu de paille lors d'un méga tsunami. Un fois mis à l'eau, les 
hauts calmes qu'ils couvent engendrent également des écosystèmes marins fertiles, dynamiques et complexes. 4. Par les temps qui courent ces sacs emplis de sable évoquent ceux où s'amassent les matières radioactives

\section{Références}

Anders, G. 2008 Le Temps de la fin. Paris: L'Herne. Bataille, G. 1967 (1949) La Part maudite. Paris: Minuit.

Barthes, R. 2002 Le Neutre. Cours au Collège de France (1977-1978). Paris: Seuil.

Berque, A. 2014 Poétique de la Terre. Histoire naturelle et histoire humaine, essai de mésologie. Paris: Belin.

— 2000 Écoumène. Introduction aux milieux humains. Paris: Belin.

Canguilhem, G. 2006 (1943) Le normal et le pathologique. Paris: PUF.

Chateauraynaud, F. 2011 Argumenter dans un champ de forces. Essai de balistique sociologique. Paris: Pétra.

Chouzenoux, A., Gorgerat, F. \& Y. Moreau 2013 Médée/ Fukushima, pièce de théâtre. Ciejours tranquilles, Lausanne.

Descola, P. 2005 Par-delà nature et culture. Paris: Gallimard.

Despret, V. 2015 Au bonheur des morts. Récits de ceux qui restent. Paris: Les empêcheurs de penser en rond / La Découverte.

Douglas, M. 1971 De la souillure. Essai sur les notions de pollution et de tabou. Paris: Maspéro.

Foucault, M. 1972 Histoire de la folie à l'âge classique. Paris: Gallimard.

Gibson, J.J. 1979 The Ecological Approach to Visual Perception. Boston: Houghton \& Miflin.

Glowczewski, B \& A. Soucaille dir. 2011 Cahiers d'anthropologie sociale 7 Désastres.

Guy, H., Jeanjean, A. \& A. Richier dir. 2014 Techniques ECulture 60 Le cadavre en procès.

Hartog, F. 2003 Régimes d'historicité. Présentisme et expériences du temps. Paris: Seuil.

Honneth, A. 2006 La société du mépris. Paris: La Découverte. produites par Fukushima Daiichi. Mais il n'en est rien. Ceux-ci servent simplement à soutenir le ciel. @ Kyoko Maruyama. Texte: Yoann Moreau.

5 et 6 . () Mathias Crémadez \& Yoann Moreau.

Husserl, E. 1989 (1934) La Terre ne se meut pas. Paris: Minuit.

Jankélévitch, V. 1986 L'imprescriptible. Paris: Seuil.

Laplantine F. 2016 Non. Négation, négatif, négativité entre Chine, Japon et Europe. Saint-Vincent-de-Mercuze: De l'incidence éditeur.

Leroi-Gourhan, A. 1964 Le geste et la parole (vol. 2). Paris: Albin Michel.

Lévi-Strauss, C. 1962 La pensée sauvage. Paris: Plon.

Montaigne, M. de 1979 (1588) Essais (vol. 3). Paris: Flammarion: 1-21.

Moreau, Y. 2014 «Approche anthropologique de la vulnérabilité et des aléas majeurs » in A. Monaco \& P. Prouzet dir. Risques côtiers et adaptation des sociétés. Londres: ISTE éditions.

- 2013 Catastrophes et mondes. Disputes et trajectoires du sens des aléas majeurs. Thèse d'anthropologie de l'EHESS, Paris (publication en cours).

Reitz, M. 2011 Je n'ai rien vu à Tchernobyl. Le difficile traitement de la mémoire et de l'histoire de la catastrophe en Ukraine. Neuchâtel: Ethnoscope.

Revet, S. 2007 Anthropologie d'une catastrophe. Les coulées de boue de 1999 au Vénézuéla. Paris: Presses Sorbonne Nouvelle.

Rey, A. 2004 «Entre calcul et expérience», Science et Avenir, hors-série 141 Les 3 constantes de l'univers décembre: 12-13.

Ricœur, P. 2000 La mémoire, l'histoire, l'oubli. Paris: Seuil. Souffron, V. dir. 2015 Communications 97 Chairs disparues.

Uexküll, J. von \& G. Kriszat 1956 (1934) Mondes animaux et monde humain. Hambourg: Gonthier.

Zarader, M. 2001 L'être et le neutre. À partir de Maurice Blanchot. Paris: Verdier.

Zonabend, F. 1989 La presqu'̂̂le au nucléaire. Paris: Odile Jacob.

\section{Pour citer cet article}

Moreau, Y. 2016 «Être en reste face aux résidus nucléaires», TechniquesECulture 65-66 «Réparer le monde. Excès, reste et innovation», p. 92-109. 